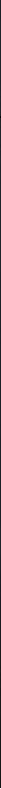

\title{
Feiner Filter für die Informationsflut
}

Fragt man deutsche Gastroenterologen ganz offiziell, welche Zeitschriften sie regelmäßig lesen, so gehen natürlich vollmundig mindestens zwei prominente englischsprachige und auch ein deutschsprachiges Journal über die Lippen. Ist man mit dem jeweiligen Kollegen vertrauter, so werden auch einige Webseiten und Printmedien genannt, die weniger den wissenschaftlichen Anspruch als die EBM- und GOÄ-Klaviatur bedienen. Die Kunst geht schließlich nach Brot. Worüber aber alle stöhnen ist die zunehmende Informationsflut, die eine wachsende Gruppe von Kollegen in den Bereich der resignierenden Perzeptionsverweigerung zu treiben droht. Wer soll beispielsweise Schritt halten mit der rasanten Entwicklung bei der Behandlung chronisch entzündlicher Darmerkrankungen oder der Hepatitis C, die uns mindestens im Halbjahrestakt ein neues „-ab “ oder „-vir“ in wechselnden Kombinationen offeriert? Von der Onkologie nicht zu reden. Aber hier einfach „Haltet ein!“ zu rufen ist auch keine Lösung. Genau da will GASTRO NEWS mit der jetzt neu aufgelegten Print-Ausgabe ansetzen. Unsere Antwort auf die Bewältigung der Informationsfülle sind knappe und von Experten kommentierte Literaturreferate, Übersichtsbeiträge mit praxisrelevanten Informationen in den einzelnen Indikationen und Berichte von den wesentlichen Kongressen der Fachgesellschaft. Epidemiologie, Dynamik des medizinischen Fortschritts und praktische Relevanz bestimmen die Themenauswahl. Dementsprechend dürfen Sie im nächsten Jahr dicht gepackte, sorgfältig ausgewählte und qualifiziert kommentierte Informationen zu den CED, zur Hepatitis C, der gastrointestinalen Onkologie, zum Reizdarm, Reflux, NASH, Leberzirrhose mit und ohne hepatische Enzephalopathie und zu den Magen-Darm-Infektionen erwarten.

GASTRONEWS wird sechsmal jährlich erscheinen. Ein hochkarätiges Herausgeberkollegium, gut gemischt aus wissenschaftlich tätigen und an der Front der Praxis arbeitenden Kollegen, garantiert die Qualität der Inhalte und ordnet die wissenschaftliche Literatur für die Bedürfnisse der Praxis ein. Dabei wollen wir möglichst wenig akademisches Lockendrehen auf der Glatze, sondern handfeste Informationen, die uns helfen, die Betreuung unserer Patienten zu optimieren und unsere eigene Arbeit zu erleichtern. Lassen Sie sich ein auf ein intensives Leseerlebnis, das Sie nicht viel Zeit kostet, aber mit fein gefilterten relevanten Informationen versorgt.

Hermann S. FüeßI, Gerald Klose, Joachim Labenz, Martin Storr

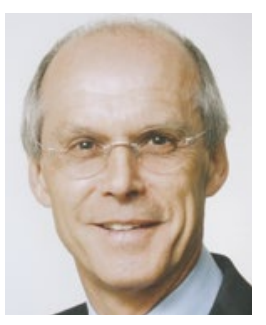

Prof. Dr. med. Hermann S. Füeßl München

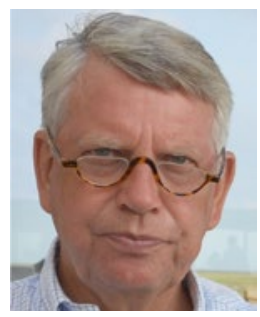

Prof. Dr. med. Gerald Klose Bremen

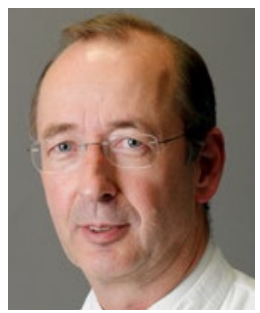

Prof. Dr. med. Joachim Labenz Siegen

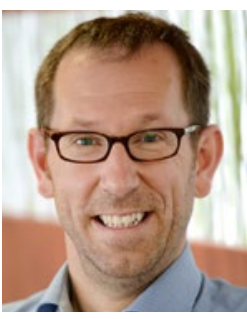

Prof. Dr. med. Martin Storr Starnberg 